\title{
Future Directions in Nutrition Research
}

\author{
Jing X. Kang \\ Massachusetts General Hospital and Harvard Medical School, Boston, Mass., USA
}

Although nutrition is generally regarded as important for human health, it is rarely utilized as a key solution for the chronic diseases that have become a global burden. Is nutrition an ineffective strategy for disease management, or is it that we have yet to demonstrate the practical use of nutrition? To date, the persuasiveness of nutrition research has been hindered by inconsistent findings, contradictory messages, and unproven effectiveness. As a result, nutrition is still not taken quite seriously by the public, and its applications have not been sufficiently implemented in clinical settings. In order to change the mainstream perception of nutrition, we must reconsider our research approach as we move forward in the field. Let us ask ourselves: what can nutrition do for today's health epidemics?

The main challenge of nutrition research lies in its complexity and confounding factors. Unlike pharmaceutical research and drug trials, many factors in our diet and lifestyle can affect the outcomes of nutritional studies, making it difficult to interpret the findings. Large, long-term studies in humans are usually viewed as the gold standard in medical research, and their results often attract media attention and have a greater influence on regulatory policies. However, these types of nutritional studies are especially prone to the confounding factors introduced by the difficulties of long-term compliance and the unavoidable changes in lifestyle over time, such as dietary and behavioral choices. In addition, current nutritional studies inherently suffer from variations in the standards for test materials (e.g., nutritional supplement ingredients), as well as in methodology, reporting, baseline evaluation, and outcome measures. Many studies have also failed to take genetic variation in study subjects into account, even though it is now well-known that this significantly affects individuals' response to dietary factors. From a translational research standpoint, nutritional findings cannot be readily implemented in clinical settings due to the lack of established biomarkers for evaluating the efficacy of nutritional interventions. In this context, it is essential that we develop new research approaches to demonstrate the potential of nutrition in healthcare.

It is my opinion that nutrition research should devote greater effort towards interventional nutrition, in addition to its traditional emphasis on epidemiological and population nutrition. In other words, we should develop nutritional interventions as solutions for the health problems we are facing worldwide. I propose the use of a pathway-based, biomarkerguided, and personalized approach to create integrated metabolic interventions. 
Dysregulated metabolism is recognized today as a critical factor in many chronic diseases. Identifying the underlying metabolic pathways of disease and developing strategies to disrupt and correct these pathways could provide a promising new approach to disease management. Given that nutrients not only serve as substrates and products for the enzymatic reactions that determine metabolism, but can also act as regulators of gene expression, it is conceivable that they could also play a key role in modulating the metabolic pathways of disease. We could therefore develop nutritional formulas that are targeted towards altering particular metabolic pathways in order to prevent and treat diseases. For example, metabolic dysregulation is one of the hallmarks of cancer [1]. Cancer cells preferentially utilize certain nutrients (e.g., glucose and glutamate) for fuel, and upregulate the lipid metabolism to produce lipid mediators (e.g., eicosanoids) to create a tumor-supporting microenvironment [2, 3]. By using multiple nutrients to modulate these pathways and interfere with the metabolic needs of cancer, nutritional interventions are capable of inhibiting cancer development. In fact, many natural compounds have been shown to have anticancer effects, and would most likely be good candidates for use in nutritional interventions. Furthermore, compared to drugs, nutrients are safe and can be readily combined to be effective for multiple targets. The efficacy of nutritional interventions can therefore be highlighted by the use of appropriate nutrients that target specific metabolic pathways to restore health conditions.

The alteration of a metabolic pathway can result in changes in the metabolite profile, functional processes, and the symptoms of a disease, which serve as biomarkers of the pathway. Identifying the relevant biomarkers and establishing reliable methods of measurement should allow us to better evaluate the effectiveness of an intervention. For example, the cyclooxygenase-2 (COX-2) pathway of lipid metabolism can be targeted to suppress an inflammatory response. A nutrient that can interfere with COX-2 activity can be expected to lower the levels of the metabolite prostaglandin $E_{2}$, leading to the downregulation of inflammatory factors, such as the transcriptional factor nuclear factor kappa B

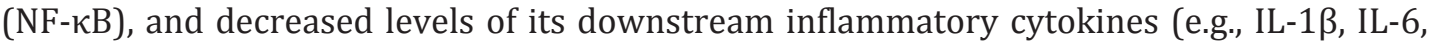
and TNF- $\alpha$ ) [4]. Currently, well-defined biomarkers for establishing the metabolic effects of nutrients are still lacking; future studies that utilize cutting-edge technologies (such as the 'omics') are needed to identify biomarkers that are sensitive and can be readily assessed. Ultimately, the testing of these biomarkers will help physicians and patients select a suitable nutritional intervention, monitor its efficacy and progress, and tailor their subsequent treatment.

Individual variation is now known to cause great variability in health outcomes. Nutritional studies attempt to control for factors such as age, sex, weight, and exercise, but two major factors that remain largely unaccounted for are genetic variation and the gut microbiota. Gene variants and gut microbial composition can significantly influence how an individual responds to certain dietary factors. For example, polymorphisms in the fatty acid desaturase (FADS) gene cluster have been linked to significant variability in response to omega-3 fatty acid supplementation [5] and could even contribute to health disparities between populations, such as diabetes or obesity [6]. The gut microbiota have been shown to vary greatly among individuals (due to factors such as diet, age, sex, ethnicity, and geographic location), which can then differentially impact the digestion, absorption, and metabolism of nutrients [7]. The intervention dose and duration needed to achieve significant effects could therefore be markedly different among groups or individuals. The failure to account for such interindividual variability may introduce confounding factors that lead to confusing results. Future research to investigate the interaction between genetic variation and metabolism the subject matter of nutrigenetics - and to elucidate the role of the gut microbiota in metabolism should help us to properly design and interpret nutritional studies. 
The potential of interventional nutrition as a solution for health problems may thus be realized using a pathway-based, biomarker-guided, and personalized approach. The fields of nutrigenetics and nutrigenomics are well equipped to accelerate the development of these integrated metabolic interventions. Advances in interventional nutrition research may not only improve the public and professional perception of nutrition, but also provide more options for the management of modern chronic diseases.

\section{References}

- 1 Hanahan D, Weinberg RA: Hallmarks of cancer: the next generation. Cell 2011;144:646-674.

- 2 Wang D, DuBois RN: Eicosanoids and cancer. Nat Rev Cancer 2010;10:181-193.

- 3 He C, Qu X, Wan J, Rong R, Huang L, Cai C, Zhou K, Gu Y, Qian SY, Kang JX: Inhibiting delta-6 desaturase activity suppresses tumor growth in mice. PLoS One 2012;7:e47567.

4 Kang JX, Weylandt KH: Modulation of inflammatory cytokines by omega-3 fatty acids. Subcell Biochem 2008; 49:133-143.

5 Simopoulos AP: Genetic variants in the metabolism of omega- 6 and omega- 3 fatty acids: their role in the determination of nutritional requirements and chronic disease risk. Exp Biol Med (Maywood) 2010;235:785-795.

6 Sergeant S, Hugenschmidt CE, Rudock ME, Ziegler JT, Ivester P, Ainsworth HC, Vaidya D, Case LD, Langefeld CD, Freedman BI, Bowden DW, Mathias RA, Chilton FH: Differences in arachidonic acid levels and fatty acid desaturase (FADS) gene variants in African Americans and European Americans with diabetes or the metabolic syndrome. Br J Nutr 2012;107:547-555.

7 Flint HJ, Scott KP, Louis P, Duncan SH: The role of the gut microbiota in nutrition and health. Nat Rev Gastroenterol Hepatol 2012;9:577-589. 